\title{
„MITTEILUNGEN DER VEREINIGUNG ÖSTERREICHISCHER BIBLIOTHEKARINNEN UND BIBLIOTHEKARE“ - ONLINEFIRST
}

Liebe Leserin, lieber Leser,

wir freuen uns, Ihnen mitteilen zu können, dass seit Ausgabe 2/2019 auch bei den Mitteilungen der VÖB das Konzept von OnlineFirst umgesetzt wird. Das bedeutet, dass jeder Beitrag unmittelbar nach dem Begutachtungsund Layoutierungsprozess online veröffentlicht wird, sodass die einzelnen Artikel unabhängig vom Erscheinungstermin der jeweiligen (Print-)Ausgabe online zugänglich und somit top-aktuell verfügbar sind. So erschienen die Beiträge der vorliegenden Ausgabe 1/2020 OnlineFirst zwischen Jänner und April 2020. Wir hoffen, dass dieses Konzept für Autorinnen und Autoren sowie für Leserinnen und Leser gleichermaßen attraktiv ist und die Akzeptanz der Mitteilungen der VÖB als wichtige Informationsplattform noch weiter steigern wird.

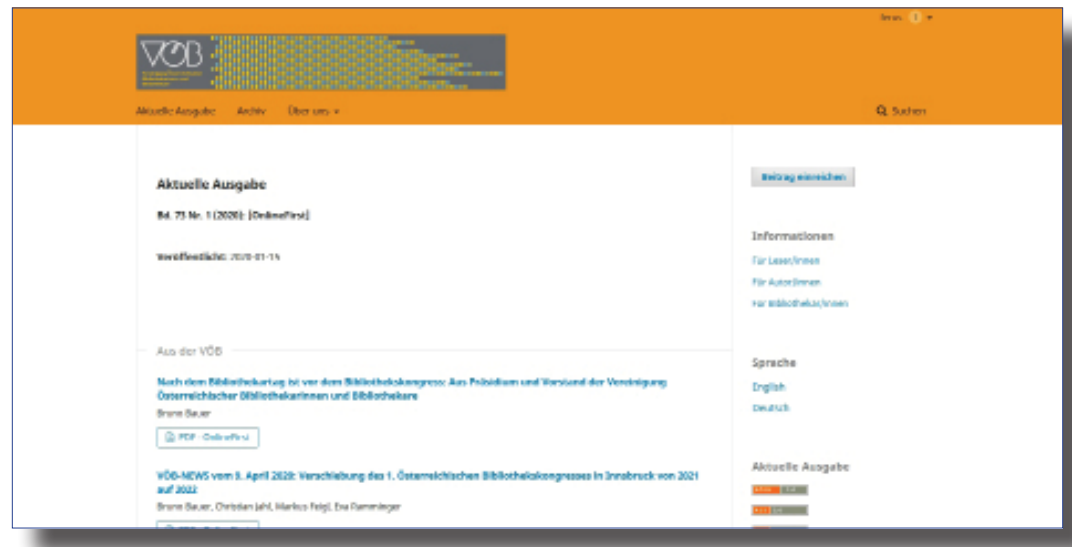

Welche Beiträge, Reports, Mitteilungen, Berichte und Rezensionen sowie Informationen aus der Vereinigung Österreichischer Bibliothekarinnen und Bibliothekare erwarten Sie in der aktuellen Ausgabe?

\section{Aus der VÖB}

Unter dem Titel „Nach dem Bibliothekartag ist vor dem Bibliothekskongress: Aus Präsidium und Vorstand der Vereinigung Österreichischer Bibliothekarinnen 
und Bibliothekare" legt Bruno Bauer, der im September 2019 neu gewählte Präsident der Vereinigung Österreichischer Bibliothekarinnen und Bibliothekare, dar, welche Schwerpunkte die kommende Funktionsperiode der VÖB (2019-2021) prägen werden [S. 10].

In Ergänzung dazu wird die Erklärung der Verantwortlichen der VÖB, des Büchereiverbandes Österreich (BVÖ) sowie der Universitäts- und Landesbibliothek Tirol (ULBT) abgedruckt, in der die durch die Corona-Krise bedingte Verschiebung des 1. Österreichischen Bibliothekskongresses in Innsbruck von 2021 auf 2022 begründet wird [S. 20].

Extra hingewiesen wird auch noch einmal auf die von Harald Weigel, dem langjährigen Präsidenten der VÖB, herausgegebenen „Schriften der Vereinigung Österreichischer Bibliothekarinnen und Bibliothekare (VÖB)", in der zwischen 2007 und 2015 insgesamt 14 Bände, gedruckt beim Wolfgang Neugebauer Verlag, erschienen sind. Sämtliche Bände sind, z.T. auch Print-on-Demand, lieferbar [S. 21].

Susanne Blumesberger informiert über die „Gründung der VÖB-AG Barrierefreiheit in Bibliotheken", und deren Zielsetzung [S. 29].

Weiters wird das von Markus Lackner erstellte „Protokoll der 64. Generalversammlung der Vereinigung österreichischer Bibliothekarinnen und Bibliothekare", die im Rahmen des 34. Österreichischen Bibliothekartages am 12. September 2019 in Graz stattgefunden hat, veröffentlicht [S. 30].

\section{Beiträge}

„NICHT nicht lesen. Ein bibliothekarischer Journal Club an der Universitätsbibliothek Wien" - unter diesem Titel berichten Stefan Alker-Windbichler und Benedikt Lodes über die Konzeption und Etablierung eines Journal Clubs an der Universitätsbibliothek Wien. Dieses innovative Fortbildungsangebot besteht aus monatlich stattfindenden Treffen, in denen vorab gelesene bibliothekarische Fachliteratur in einem strukturierten Rahmen vorgestellt und besprochen werden [S. 35].

Doris Ernst, Gertraud Novotny und Eva Maria Schönher informieren in ihrem Beitrag „(Core Trust) Seal your repository!“ über die Tätigkeit einer im Rahmen des Netzwerks für RepositorienmanagerInnen (RepManNet) entstandenen Arbeitsgruppe, die sich dem Ziel der Zertifizierung von Repositorien in Österreich widmet und die Entwicklung einer „DINI-Zertifizierung in Österreich" anstrebt [S. 46].

Unter dem Titel „Der Umbau der Universitätsbibliothek Graz - ein Bericht in drei Fortsetzungen. Teil 3: Die unvollendete Vollendung" gibt Werner Schlacher einen Einblick in die letzte Etappe des Umbaus - von der Gleichenfeier im 
Juli 2018 über die Eröffnung des Gebäudes im September 2019 bis zur Inbetriebnahme der UB Graz [S. 60].

\section{Reports}

Von Thomas Haselwanter wurde der Report „e-Infrastructures Austria Plus. Kurzfassung des Abschlussberichts über das HRSM-Projekt für das Schaffen, Ansiedeln und Vernetzen von Infrastruktur zum Erstellen und Verwalten von Projektanträgen und Forschungsdaten" vorgelegt, der einen Einblick in die Umsetzung des vom BMBWF zwischen 2017 und 2019 geförderten Hochschulraumstrukturmittelprojektes e-Infrastructures Austria Plus bietet, in dessen Rahmen acht Arbeitspakete bearbeitet wurden (Research Lifecycle; Policy zum FDM; Datenmanagementpläne; Institutionelle Repositorien; Metadaten; Persistente Identifikatoren; GO FAIR; eLab Notebooks) [S. 71].

Birgit Hörzer, Gabriele Pum, Alina Rezniczek und Monika Schneider-Jakob informieren unter dem Titel „Neues aus der bibliothekarischen Aus- und Fortbildung in den Studienjahren 2018/19 und 2019/20" über die Aktualisierung des Curriculums für Grund- und Masterlehrgang des ULG Library and Information Studies, über die Grundlehrgänge 2018/19 und 2019-2021, den Masterlehrgang 2019-2021 sowie die Abschlüsse 2018 und 2019. Vorgestellt werden auch in Form der Abstracts die Masterarbeiten 2019 sowie das österreichweite praxisnahe Fortbildungsprogramm 4L - LifeLong Learning Librarians [S. 118].

\section{Mitteilungen}

Mathis Kronschläger berichtet unter dem Titel „Mitteilungen der OBVSG“ über aktuelle Ereignisse aus dem Verbund und aus der Verbundzentrale. Er informiert über die Verbundsuchmaschine Primo, den Verbundkatalog Alma, die Nutzung des Digitalen Assistenten für die Sacherschließung, über die Langzeitarchivierung und die Gemeinsame Normdatei [S. 127].

\section{Berichte}

Unter dem Titel „Als Informationsspezialistln in die Selbstständigkeit?“ informiert Birgit Bauer über ihre Teilnahme an der AIIP-Konferenz, die von 11. bis 14 . April 2019 in Philadelphia stattgefunden hat [S. 134].

Eindrücke von der Enquete „Nationalsozialismus digital - Die Verantwortung von Bibliotheken, Archiven und Museen sowie Forschungseinrichtungen und Medien im Umgang mit der NS-Zeit im Netz", die von der Österreichischen Nationalbibliothek und vom Institut für Zeitgeschichte der Universität Wien von 
27. bis 29. November 2019 in Wien veranstaltet wurde, bieten Barbara Kern-Flora und Justus Düren in ihrem Bericht [S. 141].

Unter dem Titel „Ausgewählte Erkenntnisse aus der Enquete Nationalsozialismus digital" fasst Markus Stumpf, einer der drei Organisatoren dieser Veranstaltung, wichtige Aspekte, die im Zusammenhang mit der Digitalisierung nationalsozialistischer Quellen zu beachten sind, zusammen [S. 147].

Eine Perspektive von außen auf diese Veranstaltung bietet der Beitrag „Aus dem Giftschrank in das Internet? Ist Aufklärung über NS-Propaganda im offenen Wissenschaftsnetz möglich? Eine Tagung in Wien zur Verantwortung von Bibliotheken und Museen sucht nach neuen Wegen", den Thomas Bürger, Generaldirektor a.D. der Sächsischen Landesbibliothek - Staats- und Universitätsbibliothek Dresden (SLUB), verfasst hat [S. 152].

\section{Rezensionen}

In der aktuellen Ausgabe der Mitteilungen der VÖB werden fünf aktuelle Publikationen vorgestellt; die betreffenden Rezensionen wurden verfasst von Bruno Bauer (Lexikon der österreichischen Provenienzforschung - https://www.lexikon-provenienzforschung.org/ [S. 158]), Constantin Cazan (Elke Lang \& Andreas Bohne-Lang: Praxishandbuch IT-Grundlagen für Bibliothekare [S. 167]), Lorenz Mikoletzky [Ilse Korotin \& Edith Stumpf-Fischer (Hg.): Bibliothekarinnen in und aus Österreich [S. 176] und Josef Pauser (Gedeon Borsa: Catalogus librorum sedecimo saeculo impressorum qui in Bibliotheca Nationali Austriae asservantur / Katalog der Drucke des 16. Jahrhunderts in der Österreichischen Nationalbibliothek XIII-XIV [S. 178]; Ludger Syré (Hrsg.): Ressourcen für die Forschung. Spezialsammlungen in Regionalbibliotheken [S. 184]).

Wir wünschen ihnen viel Spaß und die eine oder andere Anregung beim Lesen und freuen uns über Ihr Feedback.

Ihr Editorial Board der Mitteilungen der VÖB

DOI: https://doi.org/10.31263/voebm.v73i1.3607

(c) Editorial Board der Mitteilungen der VÖB

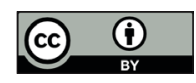

Dieses Werk ist - exkl. einzelner Logos und Abbildungen - lizenziert unter einer Creative-Commons-Lizenz Namensnennung 4.0 International 